\title{
Decomposition theorem for the cd-index of Gorenstein* posets
}

\author{
Richard Ehrenborg • Kalle Karu
}

Received: 20 June 2006 / Accepted: 15 December 2006 /

Published online: 13 January 2007

(C) Springer Science + Business Media, LLC 2007

\begin{abstract}
We prove a decomposition theorem for the cd-index of a Gorenstein* poset analogous to the decomposition theorem for the intersection cohomology of a toric variety. From this we settle a conjecture of Stanley that the cd-index of Gorenstein* lattices is minimized on Boolean algebras.
\end{abstract}

Keywords cd-index · Gorenstein* posets · Decomposition theorem · Lattices · Subdivisions

\section{Introduction}

The cd-index of a convex polytope $P$ is a polynomial $\Psi_{P}(\mathbf{c}, \mathbf{d})$ in the non-commuting variables $\mathbf{c}$ and $\mathbf{d}$ that effectively encodes the flag $f$-vector of the polytope $P[3,4]$. Its coefficients are non-negative integers [12].

The following result was proved in [5] and used to study the monotonicity property of the cd-index:

Theorem 1.1 (Billera, Ehrenborg). For any polytope $P$ and a proper face $F$ of the polytope $P$ the coefficientwise inequality

$$
\Psi_{P} \geq \Psi_{F} \cdot \Psi_{\mathrm{Pyr}(P / F)}
$$

holds, where $\operatorname{Pyr}(P / F)$ is the pyramid over the polytope $P / F$.

\footnotetext{
R. Ehrenborg

Department of Mathematics, University of Kentucky, Lexington, KY 40506, USA

e-mail: jrge@ms.uky.edu

K. Karu $(\bowtie)$

Department of Mathematics, University of British Columbia, 1984 Mathematics Road, Vancouver,

B.C. Canada V6T 1 Z2

e-mail: karu@math.ubc.ca
} 
By iterating this theorem it implies that among all polytopes of dimension $n$ the cd-index is minimized by the $n$-dimensional simplex.

One can define the cd-index more generally for Eulerian posets. It has non-negative coefficients if the poset is Gorenstein* [10]. We generalize Theorem 1.1 to the case of Gorenstein* lattices:

Theorem 1.2. For any Gorenstein $*$ lattice $\Lambda$ and an element $v \in \Lambda$ such that $\widehat{0}<$ $v<\widehat{1}$ the coefficientwise inequality

$$
\Psi_{\Lambda} \geq \Psi_{[\widehat{0}, \nu)} \cdot \Psi_{\operatorname{Pyr}[v, \widehat{1})}
$$

holds, where $\operatorname{Pyr}[v, \widehat{1})$ is the pyramid over the poset $[v, \widehat{1})$.

The use of half-open intervals in the statement of the theorem is explained in the next section. Considering the dual lattice $\Lambda^{*}$, we obtain by duality

$$
\Psi_{\Lambda} \geq \Psi_{\mathrm{Pyr}[\widehat{0}, v)} \cdot \Psi_{[v, \widehat{1})} .
$$

The action of taking the pyramid of an Eulerian poset on the cd-index is described by the following linear map. Let $G$ be a derivation on $\mathbb{Z}\langle\mathbf{c}, \mathbf{d}\rangle$ defined by $G(\mathbf{c})=\mathbf{d}$ and $G(\mathbf{d})=\mathbf{c d}$. Next let Pyr be the operator on $\mathbb{Z}\langle\mathbf{c}, \mathbf{d}\rangle$ defined by $\operatorname{Pyr}(w)=w \cdot \mathbf{c}+G(w)$. Then the cd-index of the pyramid is the pyramid of the cd-index, that is, $\Psi_{\operatorname{Pyr}(P)}=$ $\operatorname{Pyr}\left(\Psi_{P}\right)$; see [8].

As an example, if $\Lambda$ is the face lattice of a plane $n$-gon and $v$ is a vertex, then the inequality of Theorem 1.2 reads

$$
\mathbf{c}^{2}+(n-2) \mathbf{d} \geq \mathbf{c}^{2}+\mathbf{d} .
$$

To see that the theorem does not extend to Gorenstein* posets that are not lattices, take $\Lambda$ to be the poset of the plane 2-gon, that is, the $C W$-complex consisting of two edges glued at the two endpoints. The cells of this complex form a Gorenstein* poset, but the inequality above with $n=2$ does not hold.

As a corollary to Theorem 1.2 we settle a conjecture of Stanley:

Corollary 1.3. Among all Gorenstein* lattices the cd-index is minimized by the Boolean algebra.

Recall that to a polytope $P$ one can associate its $h$-polynomial $h_{P}(t)$ and its $g$-polynomial $g_{P}(t)$. The inequality in Theorem 1.1 was motivated by a similar inequality between the $g$-polynomials conjectured by Kalai and proved in the case of rational polytopes by Braden and MacPherson [6].

Theorem 1.4 (Braden, MacPherson). For any rational polytope $P$ and a proper face $F$ of $P$ the following inequality holds coefficientwise:

$$
g_{P} \geq g_{F} \cdot g_{P / F}
$$


Since $g_{P / F}=g_{\operatorname{Pyr}(P / F)}$, the two inequalities in Theorems 1.1 and 1.4 can be made to look even more similar.

Theorem 1.4 was generalized to the case of nonrational polytopes in [2,7], subject to the assumption that the $g$-polynomials involved are non-negative [9]. This generalization is proved using a combinatorial decomposition theorem for pure sheaves on a fan: a pure (i.e., locally free and flabby) sheaf on a fan decomposes into elementary sheaves. Another consequence of this combinatorial decomposition theorem is the monotonicity of the $h$-vector under subdivisions: if $\widehat{\Delta}$ is a subdivision of a complete fan $\Delta$ then

$$
h_{\widehat{\Delta}} \geq h_{\Delta}
$$

coefficientwise.

We prove an analogous decomposition theorem for the cd-index. A more precise statement is given in Section 2.

Theorem 1.5. Let $\widehat{\Pi}$ be a subdivision of a Gorenstein* poset $\Pi$. Then the following inequality holds coefficientwise:

$$
\Psi_{\widehat{\Pi}} \geq \Psi_{\Pi}
$$

The crucial point in the last theorem is the correct definition of "subdivision". It not only includes the usual subdivisions of polyhedral fans, but also more general subdivisions of $C W$-complexes. With a correct notion of subdivision in place, Theorem 1.2 follows from Theorem 1.5. The product of cd-indices corresponds to the $*$-product of posets (see Section 2.4 below for the definition of *-product):

$$
\Psi_{[\widehat{0}, x)} \cdot \Psi_{\operatorname{Pyr}[x, \widehat{1})}=\Psi_{[\widehat{0}, x) * \operatorname{Pyr}[x, \widehat{1})} .
$$

We show that the original lattice $\Lambda$ is a subdivision of $[\widehat{0}, x) * \operatorname{Pyr}[x, \widehat{1})$ and then apply Theorem 1.5.

For a polytope $P$, the construction of $F * \operatorname{Pyr}(P / F)$ is illustrated in Fig. 1. From the original polytope $P$ we keep all faces $G \leq F$ and $G>F$. Then for each $G>F$ we cap it off with a cell $G^{\prime}$ of one smaller dimension. The resulting $C W$-complex corresponds to the poset $F * \operatorname{Pyr}(P / F)$ and it admits (the boundary complex of) $P$ as its subdivision.

\section{Decomposition theorem}

We refer to $[11,13]$ for terminology about posets. Throughout we only consider posets that are finite, graded, with minimal element $\widehat{0}$, but with no maximal element $\widehat{1}$ in general. This is motivated by the poset of a fan: every fan has a minimal 

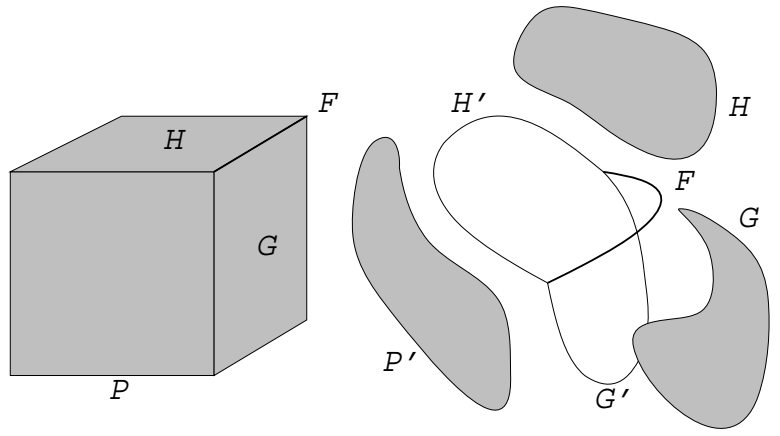

Fig. 1 Construction of $F * \operatorname{Pyr}(P / F)$ from $P$ and $F$

cone $\{0\}=\widehat{0}$, but no maximal cone in general. The rank of an element $x$ is denoted by $\rho(x)$. If $x<y$, then $\rho(x, y)=\rho(y)-\rho(x)$. We assume that the minimal element has rank $\rho(\widehat{0})=0$ and maximal elements have rank equal to the rank of the poset.

The closed (resp. half-open) intervals will be denoted $[x, y]$ (resp. $[x, y))$. If there is possibility of confusion, we write the poset $\Pi$ as the subscript: $[x, y]_{\Pi}$. Even though $\Pi$ may not contain the maximal element $\widehat{1}$, we write $[x, \widehat{1})$ for all the elements greater than or equal to $x$.

If $\Pi$ is a poset of rank $n$, let $\mathcal{O}(\Pi)$ be the order complex, that is, the set of chains in $\Pi$ ordered by inclusion.

$$
\mathcal{O}(\Pi)=\left\{\left\{\widehat{0}=\sigma_{0}<\sigma_{1}<\sigma_{2}<\cdots<\sigma_{k}\right\} \mid \sigma_{i} \in \Pi, k \geq 0\right\} .
$$

Then $\mathcal{O}(\Pi)$ is again a poset of rank $n$. In fact, $\mathcal{O}(\Pi)$ is the face poset of a simplicial complex of dimension $n-1$. (More precisely, the subsets of $\Pi$ of the form $\left\{\sigma_{1}, \sigma_{2}, \ldots, \sigma_{k}\right\}$, where $\widehat{0}<\sigma_{1}<\sigma_{2}<\cdots<\sigma_{k}$ form a simplicial complex.)

If a poset $\Pi$ is a lattice, then it obviously must have a maximal element $\widehat{1}$. If $\Pi$ does not contain $\widehat{1}$, then by abuse of terminology we say that $\Pi$ is a lattice of rank $n$ if $\Pi \cup\{\widehat{1}\}$ is a lattice of rank $n+1$. For instance, the simplicial complex $\mathcal{O}(\Pi)$ is a lattice of rank $n$ for any poset $\Pi$ of rank $n$.

A poset $\Pi$ is Eulerian if every interval satisfies the Euler-Poincaré relation. Equivalently, for any two elements $\tau, \pi$ in the poset $\Pi$ such that $\widehat{0} \leq \tau<\pi \leq \widehat{1}$, we have

$$
\sum_{\tau \leq \sigma \leq \pi}(-1)^{\rho(\tau, \sigma)}=0
$$

\subsection{Gorenstein* posets}

Definition 2.1. A simplicial complex $\Delta$ of dimension $n-1$ is called Gorenstein if it is a real homology sphere of dimension $n-1$. This means that: 
- The reduced (simplicial) homology of $\Delta$ is

$$
\widetilde{H}_{i}(\Delta, \mathbb{R})= \begin{cases}\mathbb{R} & \text { if } i=n-1 \\ 0 & \text { otherwise }\end{cases}
$$

- For every simplex $x \in \Delta$ of dimension $m$, the reduced homology of its link is

$$
\widetilde{H}_{i}\left(\operatorname{Link}_{\Delta}(x), \mathbb{R}\right)= \begin{cases}\mathbb{R} & \text { if } i=n-m-1 \\ 0 & \text { otherwise }\end{cases}
$$

A poset $\Pi$ is called Gorenstein* if its order complex $\mathcal{O}(\Pi)$ is Gorenstein.

A simplicial complex $\Delta$ is called Cohen-Macaulay if a similar condition holds, but the top degree cohomology is allowed to have any dimension. Thus a simplicial complex is Gorenstein if and only if it is Cohen-Macaulay and Eulerian. A poset $\Pi$ is called Cohen-Macaulay if its order complex $\mathcal{O}(\Pi)$ is Cohen-Macaulay. Since $\Pi$ is Eulerian if and only if $\mathcal{O}(\Pi)$ is Eulerian, it follows that a poset $\Pi$ is Gorenstein* if and only if it is Cohen-Macaulay and Eulerian.

Recall that $\operatorname{Link}_{\Delta}(x)$ is isomorphic to the interval $[x, \widehat{1})$ in $\Delta$. Thus, denoting $\operatorname{Link}_{\Delta} \widehat{(0)}=\Delta$, we see that the first homology condition in the definition is the same as the second one applied to the element $x=\widehat{0}$. Also recall that the reduced simplicial homology of $\operatorname{Link}_{\Delta}(x) \simeq[x, \widehat{1})$ for $x$ of rank $k$ is computed by the simplicial chain complex

$$
0 \longleftarrow \mathbb{R}_{x} \longleftarrow \bigoplus_{\substack{y>x \\ \rho(y)=k+1}} \mathbb{R}_{y} \longleftarrow \bigoplus_{\substack{z>x \\ \rho(z)=k+2}} \mathbb{R}_{z} \longleftarrow \cdots \longleftarrow \bigoplus_{\substack{w>x \\ \rho(w)=n}} \mathbb{R}_{w} \longleftarrow 0
$$

The homology condition in the definition of Gorenstein states that this sequence has only 1-dimensional homology at the rightmost place.

If $\Pi$ is a Gorenstein* poset of rank $n$, then any interval $[\sigma, \tau)$ in $\Pi$ is again Gorenstein*. Indeed, given a chain $x \in \mathcal{O}([\sigma, \tau))$, the interval $[x, \widehat{1})$ in $\mathcal{O}([\sigma, \tau))$ is isomorphic to the interval $[y, \widehat{1})$ in $\mathcal{O}(\Pi)$ for some $y \in \mathcal{O}(\Pi)$. Hence the homology condition for $[y, \widehat{1})$ implies the same for $[x, \widehat{1})$.

Given a Gorenstein* poset $\Pi$, its dual poset $\Pi^{*}$ (obtained by reversing the order relations) is again Gorenstein*. This follows from the fact that the two posets have isomorphic order complexes.

We also need the notion of a poset $\Pi$ such that $\mathcal{O}(\Pi)$ is a homology ball. If such an $\mathcal{O}(\Pi)$ has dimension $n-1$, then its boundary $\partial \mathcal{O}(\Pi)$ is a sub-complex of dimension $n-2$. To define the analogue for arbitrary posets, we consider pairs $(\Pi, \partial \Pi)$, where

- $\Pi$ is a poset of rank $n$;

- $\partial \Pi \subset \Pi$ is a sub-poset of $\Pi$ of rank $n-1$ (with elements in ranks $n-1$ or less);

- $\partial \Pi$ is an ideal in $\Pi$ : if $\sigma \in \partial \Pi$ and $\tau<\sigma$, then $\tau \in \partial \Pi$. 
Definition 2.2. A pair $(\Delta, \partial \Delta)$ where $\Delta$ is a simplicial complex of dimension $n-1$ is called near-Gorenstein if $\Delta$ is a real homology ball of dimension $n-1$ with boundary $\partial \Delta$. This means that:

- The complex $\partial \Delta$ is Gorenstein of dimension $n-2$.

- For every $x \in \Delta$ of dimension $m$ (including $x=\widehat{0}$ of dimension -1 ), the reduced homology of its link is

$$
\widetilde{H}_{i}\left(\operatorname{Link}_{\Delta}(x), \mathbb{R}\right)= \begin{cases}\mathbb{R} & \text { if } i=n-m-1 \text { and } x \notin \partial \Delta \\ 0 & \text { otherwise }\end{cases}
$$

A pair $(\Pi, \partial \Pi)$ is called near-Gorenstein $*$ if $(\mathcal{O}(\Pi), \mathcal{O}(\partial \Pi))$ is near-Gorenstein.

It is clear that for a near-Gorenstein* pair $(\Pi, \partial \Pi)$, the boundary $\partial \Pi$ is determined by $\Pi$. Hence we may simply say that a poset $\Pi$ is near-Gorenstein*. Similarly, we may call a simplicial complex $\Delta$ near-Gorenstein. We also denote $\operatorname{Int}(\Pi)=\Pi \backslash \partial \Pi$.

The terminology is motivated by Stanley's notion of a near-Eulerian poset [12], which is a poset obtained from an Eulerian poset of rank $n$ by removing an element of rank $n$. A similar result holds here:

Lemma 2.3. Let $\Pi$ be a Gorenstein* poset of rank $n$ and $\pi \in \Pi$ an element of rank $\rho(\pi)=n$. Then $\Pi \backslash\{\pi\}$ is a near-Gorenstein* poset of rank $n$ with boundary $[\widehat{0}, \pi)$.

Conversely, every near-Gorenstein* poset arises this way.

We will prove this lemma in Section 4.

\subsection{The cd-index}

Let us recall the combinatorial construction of the cd-index of an Eulerian poset. For a chain $x=\left\{\widehat{0}=\sigma_{0}<\sigma_{1}<\cdots<\sigma_{k}\right\}$ in a poset $\Pi$ define the weight of the chain by

$$
\operatorname{wt}(x)=(\mathbf{a}-\mathbf{b})^{\rho\left(\sigma_{0}, \sigma_{1}\right)-1} \cdot \mathbf{b} \cdot(\mathbf{a}-\mathbf{b})^{\rho\left(\sigma_{1}, \sigma_{2}\right)-1} \cdot \mathbf{b} \cdots \mathbf{b} \cdot(\mathbf{a}-\mathbf{b})^{\rho\left(\sigma_{k}, \widehat{1}\right)-1} .
$$

Here $\mathbf{a}$ and $\mathbf{b}$ are non-commuting variables of degree 1 each. Then the $\mathbf{a b}$-index of the poset $\Pi$ is given by the sum

$$
\Psi_{\Pi}=\Psi_{\Pi}(\mathbf{a}, \mathbf{b})=\sum_{x} \mathrm{wt}(x)
$$

where the sum ranges over all chains $x$ in the poset $\Pi$.

When $\Pi$ is Eulerian, it follows from the relations in [3] that $\Psi_{\Pi}(\mathbf{a}, \mathbf{b})$ can be expressed in terms of the variables $\mathbf{c}=\mathbf{a}+\mathbf{b}$ and $\mathbf{d}=\mathbf{a b}+\mathbf{b a}$. The $\mathbf{c d}$-index of $\Pi$ is the polynomial $\Psi_{\Pi}(\mathbf{a}, \mathbf{b})$ written in terms of $\mathbf{c}$ and $\mathbf{d}$. By a slight abuse of notation we 
denote the cd-index of $\Pi$ by $\Psi_{\Pi}(\mathbf{c}, \mathbf{d})$. This notation may cause concern only when we talk about non-negativity of the cd-index. By this we mean non-negativity of the coefficient of $\Psi_{\Pi}(\mathbf{c}, \mathbf{d})$ when expressed as a polynomial in $\mathbf{c}$ and $\mathbf{d}$. (The coefficients of $\Psi_{\Pi}(\mathbf{a}, \mathbf{b})$ are always non-negative.)

Note that $\Psi_{\Pi}(\mathbf{a}, \mathbf{b})$ is homogeneous of degree $n$. The same is true for $\Psi_{\Pi}(\mathbf{c}, \mathbf{d})$ where $\mathbf{c}$ has degree 1 and $\mathbf{d}$ has degree 2 .

The following non-negativity theorem proved in [10] by the second author generalizes Stanley's result of the non-negativity for the cd-index of $S$-shellable complexes [12].

Theorem 2.4. The cd-index of a Gorenstein* poset has non-negative integer coefficients.

One can also define the ab-index of a near-Gorenstein* poset $\Pi$ using the same construction. This ab-polynomial, however, cannot be expressed in terms of $\mathbf{c}$ and $\mathbf{d}$. Considering chains in the Gorenstein* poset $\Pi \cup\{\pi\}$ (Lemma 2.3), it is easy to see that:

$$
\Psi_{\Pi}(\mathbf{a}, \mathbf{b})=\Phi_{\Pi}(\mathbf{a}, \mathbf{b})+\Psi_{\partial \Pi}(\mathbf{a}, \mathbf{b}) \cdot \mathbf{a},
$$

where $\Phi_{\Pi}(\mathbf{a}, \mathbf{b})$ is a homogeneous ab-polynomial that can be expressed in terms of $\mathbf{c}$ and $\mathbf{d}$. Let us omit the multiplication with $\mathbf{a}$ at the end and define the cd-index of $\Pi$ to be

$$
\Psi_{\Pi}(\mathbf{c}, \mathbf{d}):=\Phi_{\Pi}(\mathbf{c}, \mathbf{d})+\Psi_{\partial \Pi}(\mathbf{c}, \mathbf{d}),
$$

where $\Phi_{\Pi}(\mathbf{c}, \mathbf{d})$ is the polynomial $\Phi_{\Pi}(\mathbf{a}, \mathbf{b})$ expressed in terms of $\mathbf{c}$ and $\mathbf{d}$. Note that the first summand is homogeneous of degree $n$ and the second summand is homogeneous of degree $n-1$.

The following Theorem is stated in [10] only for the case of fans. We give a generalization to the case of near-Gorenstein* posets in the appendix.

Theorem 2.5. The cd-index of a near-Gorenstein* poset has non-negative integer coefficients.

\subsection{Subdivisions}

Let us now define the notion of a subdivision.

Definition 2.6. Let $\widehat{\Pi}$ and $\Pi$ be two Gorenstein* posets of rank $n$ and let

$$
\phi: \widehat{\Pi} \rightarrow \Pi
$$

be a surjective map that preserves the order relation, but not necessarily the rank. Then $\phi$ is a subdivision if for every $\sigma \in \Pi$ the pair

$$
\left(\phi^{-1}[\widehat{0}, \sigma], \phi^{-1}[\widehat{0}, \sigma)\right)
$$


is near-Gorenstein* of rank equal to $\rho(\sigma)$.

To simplify notation, let us write $\widehat{\sigma}=\phi^{-1}[\widehat{0}, \sigma]$ and $\partial \widehat{\sigma}=\phi^{-1}[\widehat{0}, \sigma)$. Recall that by Theorem 2.5, the cd-index

$$
\Psi_{\widehat{\sigma}}=\Phi_{\widehat{\sigma}}+\Psi_{\partial \widehat{\sigma}}
$$

has all non-negative coefficients.

We can now state the decomposition theorem.

Theorem 2.7. Let $\phi: \widehat{\Pi} \rightarrow \Pi$ be a subdivision map. Then

$$
\Psi_{\widehat{\Pi}}=\sum_{\sigma \in \Pi} \Phi_{\widehat{\sigma}} \cdot \Psi_{[\sigma, \widehat{1})} .
$$

Proof: The proof of this theorem is almost entirely contained in $[2,7,10]$. It relies on the theory of pure sheaves on fans and posets. Since we will not use this theory elsewhere in this article, we will use the notation of the references above and sketch the proof.

The category of pure sheaves (i.e., locally free and flabby sheaves) on a fan $\Delta$ is semisimple: any pure sheaf $\mathcal{F}$ has a decomposition into a direct sum of simple sheaves $\mathcal{L}_{\sigma}$ indexed by cones $\sigma \in \Delta$ :

$$
\mathcal{F}=\bigoplus_{\sigma \in \Delta} V_{\sigma} \otimes \mathcal{L}_{\sigma}
$$

Here $V_{\sigma}$ is a graded vector space,

$$
V_{\sigma} \simeq \operatorname{ker}\left(\overline{\mathcal{F}_{\sigma}} \longrightarrow \overline{\mathcal{F}_{\partial \sigma}}\right)
$$

Since the map $\overline{\mathcal{F}_{\sigma}} \longrightarrow \overline{\mathcal{F}_{\partial \sigma}}$ is surjective by purity of $\mathcal{F}$, we get an equality between the Poincaré polynomials of the graded vector spaces:

$$
P_{V_{\sigma}}(t)=P_{\overline{\mathcal{F}_{\sigma}}}(t)-P_{\overline{\mathcal{F}_{\partial \sigma}}}(t)
$$

It is shown in [10] that one can define a similar theory of pure sheaves on an arbitrary poset $\Pi$, where the sheaves now are multi-graded by $\mathbb{Z}^{n}$. If $\Pi$ is Gorenstein* or nearGorenstein* then the cd-index of $\Pi$ is obtained by a change of variable formula from the multi-variable Poincaré polynomial of the module of global sections of $\mathcal{L}_{\widehat{0}}$. For $\sigma>\widehat{0}$, the sheaf $\mathcal{L}_{\sigma}$ similarly gives the cd-index of $[\sigma, \widehat{1})$.

If $\phi: \widehat{\Pi} \rightarrow \Pi$ is a subdivision map, we can decompose

$$
\phi_{*}\left(\mathcal{L}_{\widehat{0}}\right)=\bigoplus_{\sigma \in \Pi} V_{\sigma} \otimes \mathcal{L}_{\sigma}
$$


Here

$$
V_{\sigma} \simeq \operatorname{ker}\left(\overline{\mathcal{L}_{\widehat{\sigma}}} \longrightarrow \overline{\mathcal{L}_{\partial \widehat{\sigma}}}\right)
$$

Its multi-variable Poincaré polynomial is

$$
P_{V_{\sigma}}(\mathbf{t})=P_{\overline{\mathcal{L}_{\widehat{\sigma}}}}(\mathbf{t})-P_{\overline{\mathcal{L}_{\partial \widehat{\sigma}}}}(\mathbf{t})=\left(\Phi_{\widehat{\sigma}}+\Psi_{\partial \widehat{\sigma}}\right)-\Psi_{\partial \widehat{\sigma}}=\Phi_{\widehat{\sigma}},
$$

where the middle equality comes from Eq. (2.1). Now the formula in the theorem follows by taking the Poincaré polynomials of the modules of global sections on both sides of the decomposition.

Note that all of the terms in the sum of Theorem 2.7 are non-negative. Since $\Phi_{\widehat{0}}=1$, we obtain Theorem 1.5 as a corollary.

\subsection{Lattices}

Let us now deduce Theorem 1.2 from Theorem 1.5. To do this, we construct a subdivision map $\phi: \Lambda \rightarrow[\widehat{0}, v) * \operatorname{Pyr}[v, \widehat{1})$.

Given two posets $\Pi$ and $\Lambda$, define

$$
\Pi * \Lambda=\Pi \cup(\Lambda \backslash\{\widehat{0}\})
$$

with order relations being those of $\Pi$ and $\Lambda$, and additionally $\sigma<\tau$ for any $\sigma \in \Pi$ and $\tau \in \Lambda$. If $\Pi$ and $\Lambda$ have ranks $m$ and $n$, respectively, then $\Pi * \Lambda$ has rank $m+n$. The order poset $\mathcal{O}(\Pi * \Lambda)$ is the product $\mathcal{O}(\Pi) \times \mathcal{O}(\Lambda)$. It follows that if $\Pi$ and $\Lambda$ are both Gorenstein*, then so is $\Pi * \Lambda$. Also, if $\Pi$ is Gorenstein* and $\Lambda$ is near-Gorenstein*, then $\Pi * \Lambda$ is near-Gorenstein*. These two properties follow by considering tensor products of the chain complexes of $\mathcal{O}(\Pi)$ and $\mathcal{O}(\Lambda)$. Moreover, the cd-index turns the *-product into multiplication [12]:

$$
\Psi_{\Pi * \Lambda}=\Psi_{\Pi} \cdot \Psi_{\Lambda}
$$

The pyramid operation of a poset containing the maximal element $\widehat{1}$ is defined as the Cartesian product with the Boolean algebra $B_{1}=\{\widehat{0}, \widehat{1}\}$. That is, for our poset $\Pi$ we have that $\operatorname{Pyr}(\Pi) \cup\{\widehat{1}\}=(\Pi \cup\{\widehat{1}\}) \times B_{1}$. Let $P$ and $Q$ be two posets both containing maximal elements. Directly we have that if both $P$ and $Q$ are Eulerian so is their Cartesian product $P \times Q$. Similarly, if both $P$ and $Q$ are Cohen-Macaulay posets it follows from [1, Theorem 7.1] that their product $P \times Q$ is Cohen-Macaulay. Hence the class of Gorenstein* posets is closed under Cartesian product and we conclude that:

Lemma 2.8. Let $\Pi$ be a Gorenstein* poset of rank $n$. Then $\operatorname{Py}(\Pi)$ is a Gorenstein* poset of rank $n+1$. 
With notation as in Theorem 1.2, let $\Lambda$ be a Gorenstein* lattice and $v \in \Lambda, \widehat{0}<$ $v<\widehat{1}$. Define the map $\phi: \Lambda \rightarrow[\widehat{0}, v) * \operatorname{Pyr}[v, \widehat{1})$ as follows:

$$
\phi(\tau)= \begin{cases}\tau & \text { if } \tau<v \\ (\tau, \widehat{1}) & \text { if } \tau \geq v \\ (\tau \vee v, \widehat{0}) & \text { otherwise }\end{cases}
$$

Note that $\tau \vee v$ can be equal to $\widehat{1}$. In Fig. 1 the cells $G$ and $G^{\prime}$ correspond to elements $(G, \widehat{1})$ and $(G, \widehat{0})$, and the cell $P^{\prime}$ corresponds to $\left.\widehat{(1,} \widehat{0}\right)$.

We claim that $\phi$ is a subdivision map. The map $\phi$ clearly preserves the order relation. To prove surjectivity of $\phi$, the only nontrivial case is to show that $(\sigma, \widehat{0})$ lies in the image for $\sigma>v$. Replacing $\Lambda$ by the interval $[\widehat{0}, \sigma)$, we need to show that for some $\tau \in \Lambda$, we have $\tau \vee v=\widehat{1}$. If no such $\tau$ exists, then every maximal element of $\Lambda$ lies in $[v, \widehat{1})$. By descending induction on the rank of an element, and using that the lattice is Eulerian, it follows that every element must lie in $[v, \widehat{1})$. This gives a contradiction.

Finally, we need to check that the inverse images $(\widehat{\pi}, \partial \widehat{\pi})$ are near-Gorenstein* in the three cases:

(1) If $\pi=\tau<v$, then

$$
(\widehat{\pi}, \partial \widehat{\pi})=([\widehat{0}, \tau],[\widehat{0}, \tau)) .
$$

Let $\Pi$ be the Gorenstein* poset $[\widehat{0}, \tau)$. We need to show that $\Pi \cup\{\widehat{1}\}$ is nearGorenstein* with boundary $\Pi$.

(2) If $\pi=(\tau, \widehat{1})$, then we have as in the previous case

$$
(\widehat{\pi}, \partial \widehat{\pi})=([\widehat{0}, \tau],[\widehat{0}, \tau))
$$

(3) If $\pi=(\tau, \widehat{0})$, then

$$
\widehat{\pi}=[\widehat{0}, \tau) \backslash[v, \widehat{1}),
$$

with boundary

$$
\partial \widehat{\pi}=\{\sigma \in \widehat{\pi} \mid \sigma \vee v<\tau\}
$$

Let $\Pi$ be the Gorenstein* lattice $[\widehat{0}, \tau)$ containing $v$ (recall that by this we mean $\Pi \cup\{\widehat{1}\}$ is a lattice). Then we need to show that $\Pi \backslash[v, \widehat{1})$ is near-Gorenstein* with boundary

$$
\{\sigma \mid \sigma \vee v<\widehat{1}\}
$$

We state what is left to prove in the following two lemmas. 
Lemma 2.9. Let $\Pi$ be a Gorenstein* poset of rank $n$. Then $\Pi \cup\{\widehat{1}\}$ is a nearGorenstein* poset of rank $n+1$ with boundary $\Pi$.

Proof: Note that $\Pi \cup\{\widehat{1}\}$ is the $*$-product $\Pi *\{\widehat{0}, \widehat{1}\}$, where $\{\widehat{0}, \widehat{1}\}$ is near-Gorenstein* with boundary $\{\widehat{0}\}$. This implies the statement of the lemma.

Lemma 2.10. Let $\Lambda$ be a Gorenstein* lattice of rank $n$ and $v \in \Lambda \backslash \widehat{0}$. Then

$$
\Lambda \backslash[v, \widehat{1})
$$

is near-Gorenstein* of rank $n$ with boundary

$$
\{\tau \in \Lambda \backslash[v, \widehat{1}) \mid \tau \vee v<\widehat{1}\}
$$

We will prove this lemma in Section 4.

As a final remark in this section, observe that we used the join operation $\vee$ in the lattice, but not the meet operation $\wedge$. It is enough to do this since a finite join-semilattice with a minimal element 0 is a lattice; see [11, Proposition 3.3.1].

\section{Flag enumeration}

In this section we consider Eulerian posets and lattices that are not necessarily Gorenstein*. By flag enumeration we are still able to derive identities for their cdindexes.

Definition 3.1. For an Eulerian lattice $\Lambda$ with an element $v$ such that $\widehat{0}<v<\widehat{1}$ define the two subposets $\Lambda_{v}$ and $\Lambda_{v}^{\prime}$ of the lattice $\Lambda$ as follows. Let

$$
\Lambda_{v}=\{\sigma \in \Lambda \mid \sigma \vee v<\widehat{1}\}
$$

where $\Lambda_{v}$ inherits the order relations from $\Lambda$, and let $\Lambda_{v}^{\prime}$ be the semisuspension of $\Lambda_{v}$, that is,

$$
\Lambda_{v}^{\prime}=\Lambda_{v} \cup\{*\}
$$

where the new element $*$ satisfies the order relation $\sigma<*$ for all $\sigma \in \Lambda_{v}$ such that $v \not \leq \sigma$.

The geometric intuition for the poset $\Lambda_{v}$ is that it is the near-Eulerian poset consisting of all faces contained in facets which contain the face $\nu$. If $\Lambda$ is Gorenstein*, then we will show in Section 4 that $\Lambda_{v}$ is near-Gorenstein*. In fact, the posets $\Lambda \backslash[v, \widehat{1})$ of Lemma 2.10 and $\Lambda_{v}$ are complementary in that the lattice $\Lambda$ is obtained by gluing the two posets along their common boundary. 
Proposition 3.2. The ab-index of the poset $\Lambda_{v}$ is given by

$$
\Psi_{\Lambda_{\nu}}=\sum_{\nu \leq \pi<1} \Psi_{[\widehat{0}, \pi)} \cdot \mathbf{a} \cdot(\mathbf{b}-\mathbf{a})^{\rho(\pi, \widehat{1})-1} .
$$

Proof: For $\pi$ in the half open interval $[v, \widehat{1})$, let $F(\pi)$ be the sum of the weights $\operatorname{wt}(x)$ of all chains $x=\left\{\widehat{0}=\sigma_{0}<\sigma_{1}<\cdots<\sigma_{k}\right\}$ such that $\sigma_{k} \vee v=\pi$. Observe that

$$
\Psi_{[\widehat{0}, \pi)} \cdot(\mathbf{b}+(\mathbf{a}-\mathbf{b})) \cdot(\mathbf{a}-\mathbf{b})^{\rho(\pi, \widehat{1})-1}=\sum_{\nu \leq \sigma \leq \pi} F(\sigma) .
$$

Note that $\mathbf{b}+(\mathbf{a}-\mathbf{b})=\mathbf{a}$ is the result that records if the chain $x$ contains $\pi$ or not. Multiply by $(-1)^{\rho(\pi, \eta)}$ and sum over all $\pi \in[\nu, \eta]$.

$$
\begin{aligned}
\sum_{\nu \leq \pi \leq \eta} \Psi_{[0, \pi)} \cdot \mathbf{a} \cdot(\mathbf{a}-\mathbf{b})^{\rho(\pi, \widehat{1})-1} \cdot(-1)^{\rho(\pi, \eta)} & =\sum_{\nu \leq \pi \leq \eta} \sum_{\nu \leq \sigma \leq \pi} F(\sigma) \cdot(-1)^{\rho(\pi, \eta)} \\
& =\sum_{\nu \leq \sigma \leq \eta} F(\sigma) \cdot \sum_{\sigma \leq \pi \leq \eta}(-1)^{\rho(\pi, \eta)} \\
& =\sum_{\nu \leq \sigma \leq \eta} F(\sigma) \cdot \delta_{\sigma, \eta} \\
& =F(\eta) .
\end{aligned}
$$

Now the ab-index of $\Lambda_{v}$ is given by the sum

$$
\begin{aligned}
\Psi_{\Lambda_{\nu}} & =\sum_{\nu \leq \eta<\widehat{1}} F(\eta) \\
& =\sum_{\nu \leq \eta<\hat{1}} \sum_{\nu \leq \pi \leq \eta} \Psi_{[\widehat{0}, \pi)} \cdot \mathbf{a} \cdot(\mathbf{a}-\mathbf{b})^{\rho(\pi, \widehat{1})-1} \cdot(-1)^{\rho(\pi, \eta)} \\
& =\sum_{\nu \leq \pi<\widehat{1}} \Psi_{\widehat{[0, \pi)}} \cdot \mathbf{a} \cdot(\mathbf{a}-\mathbf{b})^{\rho(\pi, \widehat{1})-1} \cdot\left(\sum_{\pi \leq \eta<\hat{1}}(-1)^{\rho(\pi, \eta)}\right) \\
& =\sum_{\nu \leq \pi<\widehat{1}} \Psi_{\widehat{[0, \pi)}} \cdot \mathbf{a} \cdot(\mathbf{a}-\mathbf{b})^{\rho(\pi, \widehat{1})-1} \cdot(-1)^{\rho(\pi, \widehat{1})-1},
\end{aligned}
$$

which proves the proposition.

Proposition 3.3. The sum of the weights of all chains $x$ in $\Lambda_{v}^{\prime}$ that contain the element * is given by

$$
\sum_{\nu \leq \pi<\widehat{1}} \Psi_{[\widehat{0}, \pi)} \cdot\left((\mathbf{a}-\mathbf{b})^{\rho(\pi, \widehat{1})-1}-\mathbf{a} \cdot(\mathbf{a}-\mathbf{b})^{\rho(\pi, \widehat{1})-2} \cdot\left(1+(-1)^{\rho(\pi, \widehat{1})}\right)\right) \cdot \mathbf{b} .
$$


Proof: Assume that $\Lambda_{v}^{\prime}$ has rank $n$ and let $P$ be the poset $\Lambda_{v}^{\prime}$ with the coatoms removed. The poset $P$ can be thought of as the rank selected poset $\Lambda_{v,\{1, \ldots, n-2\}}^{\prime}$. Observe that the rank of $P$ is one less than the rank of $\Lambda_{v}^{\prime}$. However we let $\rho$ denote the rank function of $\Lambda_{v}^{\prime}$ and not that of $P$.

For $\pi$ in the poset $P$ such that $\nu \leq \pi<\widehat{1}$, let $G(\pi)$ be the sum of the weights over all chains $x=\left\{\widehat{0}=\sigma_{0}<\sigma_{1}<\cdots<\sigma_{k}\right\}$ in the poset $P$ such that $\nu \not \leq \sigma_{k}$ and $\sigma_{k} \vee v=\pi$. Similarly, let $H(\pi)$ be the sum of the weights over all chains $x$ in the poset $P$ such that $\sigma_{k} \vee v=\pi$. Observe that $H(\pi) \cdot(\mathbf{a}-\mathbf{b})=F(\pi)$, where $F(\pi)$ is the function in the proof of Proposition 3.2. Hence by Eq. (3.1) we have that

$$
H(\sigma)=\sum_{\nu \leq \tau \leq \sigma} \Psi_{[\widehat{0}, \tau)} \cdot \mathbf{a} \cdot(\mathbf{a}-\mathbf{b})^{\rho(\tau, \widehat{1})-2} \cdot(-1)^{\rho(\tau, \sigma)}
$$

Now by chain counting we have the identity

$$
\Psi_{[\widehat{0}, \pi)} \cdot(\mathbf{a}-\mathbf{b})^{\rho(\pi, \widehat{1})-1}=G(\pi)+\sum_{\nu \leq \sigma<\pi} H(\sigma)
$$

Bring $G(\pi)$ to the other side of the equation and sum over all $\pi$ such that $\nu \leq \pi<\widehat{1}$. We then obtain

$$
\begin{aligned}
& \sum_{\nu \leq \pi<\widehat{1}} G(\pi)-\sum_{\nu \leq \pi<\widehat{1}} \Psi_{\widehat{[}, \pi)} \cdot(\mathbf{a}-\mathbf{b})^{\rho(\pi, \widehat{1})-1} \\
& =-\sum_{\nu \leq \sigma<\pi<\widehat{1}} H(\sigma) \\
& =-\sum_{\nu \leq \tau \leq \sigma<\pi<\widehat{1}} \Psi_{\widehat{[0}, \tau)} \cdot \mathbf{a} \cdot(\mathbf{a}-\mathbf{b})^{\rho(\tau, \widehat{1})-2} \cdot(-1)^{\rho(\tau, \sigma)} \\
& =\sum_{\nu \leq \tau<\pi<\widehat{1}} \Psi_{[\widehat{0}, \tau)} \cdot \mathbf{a} \cdot(\mathbf{a}-\mathbf{b})^{\rho(\tau, \widehat{1})-2} \cdot(-1)^{\rho(\tau, \pi)} \\
& =-\sum_{\nu \leq \tau<\widehat{1}} \Psi_{[\widehat{0}, \tau)} \cdot \mathbf{a} \cdot(\mathbf{a}-\mathbf{b})^{\rho(\tau, \widehat{1})-2} \cdot\left(1+(-1)^{\rho(\tau, \widehat{1})}\right) .
\end{aligned}
$$

Making a change of variable in the last sum and rearranging the sums gives:

$$
\sum_{\nu \leq \pi<\widehat{1}} G(\pi)=\sum_{\nu \leq \pi<\widehat{1}} \Psi_{[\widehat{0}, \pi)} \cdot\left((\mathbf{a}-\mathbf{b})^{\rho(\pi, \widehat{1})-1}-\mathbf{a} \cdot(\mathbf{a}-\mathbf{b})^{\rho(\pi, \widehat{1})-2} \cdot\left(1+(-1)^{\rho(\pi, \widehat{1})}\right)\right)
$$

The result now follows by multiplying on the right by $\mathbf{b}$. 
Following [5] define cd-polynomials $\alpha_{n}$ by $\alpha_{0}=-1$ and otherwise by

$$
\begin{aligned}
\alpha_{2 k} & =-\frac{1}{2}\left[\left(\mathbf{c}^{2}-2 \mathbf{d}\right)^{k}+\mathbf{c} \cdot\left(\mathbf{c}^{2}-2 \mathbf{d}\right)^{k-1} \cdot \mathbf{c}\right], \\
\alpha_{2 k+1} & =\frac{1}{2}\left[\left(\mathbf{c}^{2}-2 \mathbf{d}\right)^{k} \cdot \mathbf{c}+\mathbf{c} \cdot\left(\mathbf{c}^{2}-2 \mathbf{d}\right)^{k}\right] .
\end{aligned}
$$

Lemma 3.4. The cd-polynomial $\alpha_{k}$ is given by

$$
\alpha_{k}=\mathbf{a} \cdot(\mathbf{b}-\mathbf{a})^{k-1}+\left((\mathbf{a}-\mathbf{b})^{k-1}-\mathbf{a} \cdot(\mathbf{a}-\mathbf{b})^{k-2} \cdot\left(1+(-1)^{k}\right)\right) \cdot \mathbf{b} .
$$

Proof: When $k$ is even we have

$$
\begin{aligned}
-\mathbf{a} & \cdot(\mathbf{a}-\mathbf{b})^{k-1}+(\mathbf{a}-\mathbf{b})^{k-1} \cdot \mathbf{b}-2 \cdot \mathbf{a} \cdot(\mathbf{a}-\mathbf{b})^{k-2} \cdot \mathbf{b} \\
& =-\mathbf{a} \cdot(\mathbf{a}-\mathbf{b})^{k-2} \cdot(\mathbf{a}-\mathbf{b})+(\mathbf{a}-\mathbf{b}) \cdot(\mathbf{a}-\mathbf{b})^{k-2} \cdot \mathbf{b}-2 \cdot \mathbf{a} \cdot(\mathbf{a}-\mathbf{b})^{k-2} \cdot \mathbf{b} \\
& =-\mathbf{a} \cdot(\mathbf{a}-\mathbf{b})^{k-2} \cdot \mathbf{a}-\mathbf{b} \cdot(\mathbf{a}-\mathbf{b})^{k-2} \cdot \mathbf{b} \\
& =-\frac{1}{2} \cdot\left((\mathbf{a}-\mathbf{b}) \cdot(\mathbf{a}-\mathbf{b})^{k-2} \cdot(\mathbf{a}-\mathbf{b})+(\mathbf{a}+\mathbf{b}) \cdot(\mathbf{a}-\mathbf{b})^{k-2} \cdot(\mathbf{a}+\mathbf{b})\right) \\
& =\alpha_{k} .
\end{aligned}
$$

For the odd case, begin by observing that $\mathbf{a} \cdot(\mathbf{a}-\mathbf{b})^{k-1}-\mathbf{b} \cdot(\mathbf{a}-\mathbf{b})^{k-1}=(\mathbf{a}-$ $\mathbf{b})^{k-1} \cdot \mathbf{a}-(\mathbf{a}-\mathbf{b})^{k-1} \cdot \mathbf{b}$. By rearranging the terms we have

$$
\mathbf{a} \cdot(\mathbf{a}-\mathbf{b})^{k-1}+(\mathbf{a}-\mathbf{b})^{k-1} \cdot \mathbf{b}=(\mathbf{a}-\mathbf{b})^{k-1} \cdot \mathbf{a}+\mathbf{b} \cdot(\mathbf{a}-\mathbf{b})^{k-1} .
$$

Since the two sides are equal, they are also equal to their mean value. Thus we have $\mathbf{a} \cdot(\mathbf{a}-\mathbf{b})^{k-1}+(\mathbf{a}-\mathbf{b})^{k-1} \cdot \mathbf{b}=\frac{1}{2}\left((\mathbf{a}+\mathbf{b}) \cdot(\mathbf{a}-\mathbf{b})^{k-1}+(\mathbf{a}-\mathbf{b})^{k-1} \cdot(\mathbf{a}+\mathbf{b})\right)=\alpha_{k}$.

We can now give an explicit formula for the cd-index of the poset $\Lambda_{v}^{\prime}$. This formula generalizes Corollary 4.4 in [5]. This corollary was proved using line shellings, thus restricting the results in [5] to polytopes.

Theorem 3.5. For an Eulerian lattice $\Lambda$ and an element $v, \widehat{0}<v<\widehat{1}$, the cd-index of $\Lambda_{v}^{\prime}$ is given by

$$
\Psi_{\Lambda_{v}^{\prime}}=\sum_{\nu \leq \pi<\widehat{1}} \Psi_{[\widehat{0}, \pi)} \cdot \alpha_{\rho(\pi, \widehat{1})} .
$$


Proof: Add Propositions 3.2 and 3.3 using Lemma 3.4 to simplify.

We now outline a different proof of Theorem 1.2 following the argument of the polytope case in [5]. First observe that we have the following corollary to the decomposition theorem, Theorem 1.5.

Corollary 3.6. Let $\Lambda$ be a Gorenstein* lattice and let $v$ be an element of $\Lambda$ such that $\widehat{0}<v<\widehat{1}$. Then the following coefficientwise inequality holds for the cd-indexes of $\Lambda_{v}^{\prime}$ and $\Lambda$ :

$$
\Psi_{\Lambda_{v}^{\prime}} \leq \Psi_{\Lambda}
$$

Proof: The poset $\Lambda$ is a subdivision of $\Lambda_{v}^{\prime}$ by the map $\phi: \Lambda \rightarrow \Lambda_{v}^{\prime}$ defined by

$$
\phi(\tau)= \begin{cases}\tau & \text { if } \tau \in \Lambda_{\nu} \\ * & \text { if } \tau \notin \Lambda_{\nu} .\end{cases}
$$

The map $\phi$ collapses everything outside $\Lambda_{v}$ to the element $*$. We only have to check that the inverse image of the element $[\widehat{0}, *]$ is a near Gorenstein* poset. But this is the content of Lemma 2.10 and the corollary follows from Theorem 1.5, the decomposition theorem.

Theorem 1.2 is implied by the following three statements: (1) Theorem 3.5, (2) the inequality in Corollary 3.6, and (3) the equality [5, Proposition 4.6]:

$$
\operatorname{Pyr}\left(\Psi_{[\tau, \pi)}\right)-\alpha_{\rho(\tau, \pi)}=\sum_{\tau<\sigma<\pi} \alpha_{\rho(\tau, \sigma)} \cdot \operatorname{Pyr}\left(\Psi_{[\sigma, \pi)}\right),
$$

where $[\tau, \pi]$ is an interval in an Eulerian poset. The proof is verbatim to the proof of Theorem 5.1 in [5].

\section{Sheaves on posets}

In this section we introduce sheaves on posets and prove Lemmas 2.3 and 2.10.

Definition 4.1. A sheaf $F$ on a poset $\Pi$ consists of the data

- A real vector space $F_{\sigma}$ for each $\sigma \in \Pi$, called the stalk of $F$ at $\sigma$.

- Linear maps res $\operatorname{res}_{\tau}^{\sigma}: F_{\sigma} \rightarrow F_{\tau}$ for each $\sigma>\tau$, satisfying the condition $\operatorname{res}_{v}^{\tau} \circ \operatorname{res}_{\tau}^{\sigma}=$ $\operatorname{res}_{v}^{\sigma}$ whenever $\sigma>\tau>v$. These maps are called restriction maps.

A map of sheaves $F \rightarrow G$ is a collection of linear maps $F_{\sigma} \rightarrow G_{\sigma}$ commuting with the restriction maps.

The main example of a sheaf is the constant sheaf $\mathbb{R}_{\Pi}$ with all the stalks equal to $\mathbb{R}$ and all the restriction maps equal to the identity. If $F$ is a sheaf on $\Pi$ and $S \subset \Pi$, we 
let $\left.F\right|_{S}$ (or simply $F_{S}$ ) be the sheaf obtained from $F$ by setting all stalks at elements $\sigma \in \Pi \backslash S$ equal to zero. This makes sense for subsets $S$ satisfying the property that if $\tau<\sigma$ both lie in $S$, then the interval $[\tau, \sigma]$ also lies in $S$.

For the remainder of this section we consider sheaves on simplicial complexes only. All sheaves are assumed to have finite dimensional stalks.

Definition 4.2. Let $\Delta$ be a simplicial complex of rank $n$ and $F$ a sheaf on $\Delta$. The cellular complex $C^{\bullet}(F, \Delta)$ of $F$ is the complex

$$
0 \longrightarrow C^{0} \longrightarrow C^{1} \longrightarrow \cdots \longrightarrow C^{n} \longrightarrow 0
$$

where

$$
C^{k}=\bigoplus_{\substack{x \in \Delta \\ \rho(x)=n-k}} F_{x}
$$

The maps $C^{k} \rightarrow C^{k+1}$ are defined by summing the restriction maps res ${ }_{y}^{x}$ with correct sign as in the simplicial chain complex of $\Delta$.

If $S \subset \Delta$ is a subset, we write $C^{\bullet}(F, S)$ for the complex $C^{\bullet}\left(\left.F\right|_{S}, \Delta\right)$. In other words, the terms in the complex $C^{\bullet}(F, S)$ are indexed by elements of $S$.

Note that if $F=\mathbb{R}_{\Delta}$ is the constant sheaf, then $C^{\bullet}\left(\mathbb{R}_{\Delta}, \Delta\right)$ is the simplicial chain complex of $\Delta$. In particular,

$$
H^{i}\left(C^{\bullet}\left(\mathbb{R}_{\Delta}, \Delta\right)\right)=\widetilde{H}_{n-i-1}(\Delta, \mathbb{R})
$$

Recalling Definition 2.1, we have that $\Delta$ is Gorenstein if and only if

$$
\operatorname{dim} H^{i}\left(C^{\bullet}\left(\mathbb{R}_{\Delta},[x, \widehat{1})\right)\right)= \begin{cases}1 & \text { if } i=0 \\ 0 & \text { otherwise }\end{cases}
$$

for all $x \in \Delta$.

Definition 4.3. A sheaf $F$ on $\Delta$ is called Cohen-Macaulay if

$$
H^{i}\left(C^{\bullet}(F,[x, \widehat{1}))\right)=0
$$

for all $x \in \Delta$ and $i>0 . F$ is called Gorenstein if, moreover,

$$
\operatorname{dim} H^{0}\left(C^{\bullet}(F,[x, \widehat{1}))\right)=\operatorname{dim} F_{x} .
$$

Thus, the complex $\Delta$ is Gorenstein if and only if the constant sheaf on it is Gorenstein. The definition of a Cohen-Macaulay complex [13] similarly agrees with the notion of a Cohen-Macaulay sheaf. In [10] Cohen-Macaulay sheaves were called semi-Gorenstein, but Cohen-Macaulay is a more appropriate name. 
Let $F$ be a Cohen-Macaulay sheaf on $\Delta$. For any $x<y$ we can use projection maps to define a map of sheaves $F_{[x, \widehat{1})} \rightarrow F_{[y, \widehat{1})}$. This induces a map of cellular complexes, hence a map in cohomology:

$$
H^{0}\left(C^{\bullet}(F,[x, \widehat{1}))\right) \longrightarrow H^{0}\left(C^{\bullet}(F,[y, \widehat{1}))\right)
$$

We want to assemble the degree zero cohomologies into a sheaf on $\Delta$. However, the maps (4.1) go in the wrong direction compared to restriction maps. To fix this, we take the dual vector spaces and dual maps.

Definition 4.4. Let $F$ be a Cohen-Macaulay sheaf on $\Delta$. Define the sheaf $F^{\vee}$ on $\Delta$ with the stalks

$$
F_{x}^{\vee}=H^{0}\left(C^{\bullet}(F,[x, \widehat{1}))\right)^{*}
$$

and with the restriction maps being the duals of (4.1).

It is shown in [10] that the assignment $F \mapsto F^{\vee}$ defines a contravariant functor from the category of Cohen-Macaulay sheaves to the same category. In particular, $F^{\vee}$ is again Cohen-Macaulay. Moreover, $F^{\vee \vee} \simeq F$ canonically.

Consider a short-exact sequence of sheaves on $\Delta$ :

$$
0 \longrightarrow F_{1} \longrightarrow F_{2} \longrightarrow F_{3} \longrightarrow 0
$$

From the long-exact sequences in cohomology it follows that if either $F_{1}$ and $F_{2}$ or $F_{1}$ and $F_{3}$ are Cohen-Macaulay, then so is the third sheaf and we get the short-exact dual sequence

$$
0 \longleftarrow F_{1}^{\vee} \longleftarrow F_{2}^{\vee} \longleftarrow F_{3}^{\vee} \longleftarrow 0
$$

If both $F_{2}$ and $F_{3}$ are Cohen-Macaulay, then $F_{1}$ is Cohen-Macaulay if and only if the dual map $F_{3}^{\vee} \rightarrow F_{2}^{\vee}$ is injective.

Suppose that in the exact sequence above both $F_{2}$ and $F_{3}$ are Cohen-Macaulay and $F_{1}$ is supported on a subcomplex $\partial \Delta \subset \Delta$ of $\operatorname{rank} n-1$. Then $F_{1}$ is Cohen-Macaulay as a sheaf on $\partial \Delta$ and we have the dual short exact sequence

$$
0 \longleftarrow F_{2}^{\vee} \longleftarrow F_{3}^{\vee} \longleftarrow F_{1}^{\vee} \longleftarrow 0
$$

Here $F_{1}^{\vee}$ is computed by considering $F_{1}$ as a sheaf on $\partial \Delta$, hence on $\Delta$ the stalks of the dual sheaf are obtained from cohomology of degree 1 rather than degree 0 .

We can now give a stronger characterization of Gorenstein and near-Gorenstein complexes on $\Delta$.

Lemma 4.5. A simplicial complex $\Delta$ is Gorenstein if and only if $\mathbb{R}_{\Delta}$ is CohenMacaulay and

$$
\mathbb{R}_{\Delta}^{\vee} \simeq \mathbb{R}_{\Delta}
$$


Proof: Note that by Definition 2.1 the complex $\Delta$ is Gorenstein if and only if it is Cohen-Macaulay and $\mathbb{R}_{\Delta}^{\vee}$ has one-dimensional stalks. We need to show that the restriction maps in the dual sheaf are all isomorphisms. Then via $\operatorname{res}_{\hat{0}}^{x}$ all stalks are compatibly isomorphic to $\mathbb{R}_{\Delta, \widehat{0}}^{\vee}=\mathbb{R}$.

Decompose $\Delta=U \sqcup V$, where

$$
U=\left\{x \in \Delta \mid \operatorname{res}_{0}^{x}: \mathbb{R}_{\Delta, x}^{\vee} \longrightarrow \mathbb{R}_{\Delta, \hat{0}}^{\vee} \text { is zero }\right\}
$$

Then all the restriction maps in $\mathbb{R}_{\Delta}^{\vee}$ between stalks at points lying in different sets $U$ and $V$ are zero. Hence

$$
\mathbb{R}_{\Delta}^{\vee}=\left.\left.\mathbb{R}_{\Delta}^{\vee}\right|_{U} \oplus \mathbb{R}_{\Delta}^{\vee}\right|_{V}
$$

But then computing the dual $\mathbb{R}_{\Delta}^{\vee v}=\mathbb{R}_{\Delta}$, we see that it decomposes, which is impossible because all the restriction maps in $\mathbb{R}_{\Delta}$ are the identity maps. Hence $U=\emptyset$.

Lemma 4.6. Let $\Delta$ be a simplicial complex of rank $n$ and $\partial \Delta \subset \Delta$ a subcomplex of rank $n-1$. Then $\Delta$ is near-Gorenstein with boundary $\partial \Delta$ if and only if $\mathbb{R}_{\Delta}$ is Cohen-Macaulay and

$$
\mathbb{R}_{\Delta}^{\vee} \simeq \mathbb{R}_{\operatorname{Int}(\Delta)}
$$

where $\operatorname{Int}(\Delta)=\Delta \backslash \partial \Delta$.

Proof: First we assume that the conditions given in the lemma are satisfied and show that then $\Delta$ is near-Gorenstein with the given boundary. To see that $\partial \Delta$ is Gorenstein of rank $n-1$, consider the exact sequence of sheaves

$$
0 \longrightarrow \mathbb{R}_{\partial \Delta} \longrightarrow \mathbb{R}_{\Delta} \longrightarrow \mathbb{R}_{\text {Int } \Delta} \longrightarrow 0 \text {. }
$$

Since $\mathbb{R}_{\Delta}$ and $\mathbb{R}_{\operatorname{Int}(\Delta)}$ are both Cohen-Macaulay (they are dual to each other by assumption), and $\mathbb{R}_{\partial \Delta}$ is supported on the rank $n-1$ subcomplex $\partial \Delta$, we get the dual sequence (4.2)

$$
0 \longrightarrow \mathbb{R}_{\partial \Delta}^{\vee} \longrightarrow \mathbb{R}_{\Delta} \longrightarrow \mathbb{R}_{\operatorname{Int}(\Delta)} \longrightarrow 0
$$

It follows that $\mathbb{R}_{\partial \Delta}^{\vee}=\mathbb{R}_{\partial \Delta}$ and $\partial \Delta$ is Gorenstein by the previous lemma. The remaining homology conditions of Definition 2.2 follow from the isomorphism $\mathbb{R}_{\Delta}^{\vee} \simeq \mathbb{R}_{\operatorname{Int}(\Delta)}$.

Conversely, let us assume that $\Delta$ is near-Gorenstein with boundary $\partial \Delta$. Then $\mathbb{R}_{\Delta}$ and $\mathbb{R}_{\partial \Delta}$ are Cohen-Macaulay, hence so is $\mathbb{R}_{\operatorname{Int}(\Delta)}$ by the exact sequence (4.3) above. The dual sequence is

$$
0 \longrightarrow \mathbb{R}_{\partial \Delta} \longrightarrow \mathbb{R}_{\operatorname{Int}(\Delta)}^{\vee} \longrightarrow \mathbb{R}_{\Delta}^{\vee} \longrightarrow 0
$$

The sheaf $\mathbb{R}_{\Delta}^{\vee}$ has the same stalks as $\mathbb{R}_{\operatorname{Int}(\Delta)}$, hence $\mathbb{R}_{\operatorname{Int}(\Delta)}^{\vee}$ has the same stalks as $\mathbb{R}_{\Delta}$. It suffices to prove that $\mathbb{R}_{\operatorname{Int}(\Delta)}^{\vee} \simeq \mathbb{R}_{\Delta}$. 
As in the proof of the previous lemma, if $\mathbb{R}_{\operatorname{Int}(\Delta)}^{\vee}$ is not isomorphic to $\mathbb{R}_{\Delta}$, then it decomposes, and so does $\mathbb{R}_{\operatorname{Int}(\Delta)}$ by duality. Since all the restriction maps in $\mathbb{R}_{\text {Int( } \Delta)}$ are identity maps, it follows that the poset $\operatorname{Int}(\Delta)$ decomposes into a disjoint union (of unrelated elements). But then any sheaf on $\operatorname{Int}(\Delta)$, including $\mathbb{R}_{\Delta}^{\vee}$, also decomposes accordingly, which by duality implies that $\mathbb{R}_{\Delta}$ decomposes. This is a contradiction.

\subsection{Complementary posets}

Given a poset $\Pi$ containing a pair $\left(\Pi_{1}, \partial \Pi_{1}\right)$, define the complementary pair $\left(\Pi_{2}, \partial \Pi_{2}\right)$ by $\Pi_{2}=\Pi \backslash \operatorname{Int}\left(\Pi_{1}\right)$, and $\partial \Pi_{2}=\partial \Pi_{1}$.

Proposition 4.7. Let $\Delta$ be a Gorenstein simplicial complex of rank $n$ and $\Delta_{1}$ a nearGorenstein subcomplex of the same rank and with boundary $\partial_{1}$. Then the complementary pair $\left(\Delta_{2}, \partial \Delta_{2}\right)$ is also near-Gorenstein of rank $n$.

Proof: Consider the exact sequence of sheaves

$$
0 \longrightarrow \mathbb{R}_{\Delta_{1}} \longrightarrow \mathbb{R}_{\Delta} \longrightarrow \mathbb{R}_{\operatorname{Int}\left(\Delta_{2}\right)} \longrightarrow 0
$$

Since the left two sheaves are Cohen-Macaulay, so is the rightmost sheaf. The dual sequence is

$$
0 \longleftarrow \mathbb{R}_{\operatorname{Int}\left(\Delta_{1}\right)} \longleftarrow \mathbb{R}_{\Delta} \longleftarrow \mathbb{R}_{\operatorname{Int}\left(\Delta_{2}\right)}^{\vee} \longleftarrow 0
$$

It follows that $\mathbb{R}_{\operatorname{Int}\left(\Delta_{2}\right)}^{\vee}=\mathbb{R}_{\Delta_{2}}$ and $\Delta_{2}$ is near-Gorenstein with boundary $\partial \Delta_{2}$.

Given an arbitrary poset $\Pi$ containing a pair $\left(\Pi_{1}, \partial \Pi_{1}\right)$, with the complementary pair $\left(\Pi_{2}, \partial \Pi_{2}\right)$, we want to assume that taking the order complexes of $\Pi_{1}$ and $\Pi_{2}$, we again get complementary pairs. For this it is sufficient that $\Pi_{1}$ is closed under going down: if $\sigma \in \Pi_{1}$ then $[\widehat{0}, \sigma] \subset \Pi_{1}$ and $\operatorname{Int}\left(\Pi_{1}\right)$ is closed under going up: if $\sigma \in \operatorname{Int}\left(\Pi_{1}\right)$ then $[\sigma, \widehat{1}) \subset \operatorname{Int}\left(\Pi_{1}\right)$. If $\Pi_{1}$ satisfies these conditions, then $\Pi_{2}$ also does. When considering complementary pairs, we will always assume that one pair (hence also the other) satisfies these conditions.

Corollary 4.8. Let $\Pi$ be a Gorenstein* poset of rank $n$ and $\Pi_{1}$ a near-Gorenstein* sub-poset of the same rank and with boundary $\left(\Pi_{2}, \partial \Pi_{2}\right)$ is also near-Gorenstein* of rank $n$.

Let us now prove Lemma 2.3. The poset $\Pi \backslash\{\pi\}$ is the complement of $[\widehat{0}, \pi]$ with boundary $[\widehat{0}, \pi)$. This latter pair is near-Gorenstein* by Lemma 2.9 , hence so is $\Pi \backslash\{\pi\}$.

For the second statement of the lemma, suppose that $\Pi \backslash\{\pi\}$ is near-Gorenstein* with boundary $[\widehat{0}, \pi)$. Divide $\Delta=\mathcal{O}(\Pi)$ into $\Delta_{1}=\mathcal{O}(\Pi \backslash\{\pi\})$ and its complement $\Delta_{2}=\mathcal{O}([\widehat{0}, \pi])$. Then both $\Delta_{1}$ and $\Delta_{2}$ are near-Gorenstein, hence we have an exact 
sequence of Cohen-Macaulay sheaves

$$
0 \longrightarrow \mathbb{R}_{\Delta_{1}} \longrightarrow \mathbb{R}_{\Delta} \longrightarrow \mathbb{R}_{\operatorname{Int}\left(\Delta_{2}\right)} \longrightarrow 0
$$

with dual sequence

$$
0 \longleftarrow \mathbb{R}_{\operatorname{Int}\left(\Delta_{1}\right)} \longleftarrow \mathbb{R}_{\Delta}^{\vee} \longleftarrow \mathbb{R}_{\Delta_{2}} \longleftarrow 0
$$

It follows that $\mathbb{R}_{\Delta}^{\vee}$ has all stalks one-dimensional, hence $\Delta$ is Gorenstein by Definition 2.1 .

\subsection{Lattices}

Let us now prove Lemma 2.10. We need to show that for a Gorenstein* lattice $\Lambda$ and a nonzero element $v \in \Lambda$, the poset $\Lambda_{1}=\Lambda \backslash[v, \widehat{1})$ is near-Gorenstein* with boundary

$$
\partial \Lambda_{1}=\left\{\sigma \in \Lambda_{1} \mid \sigma \vee v<\widehat{1}\right\}
$$

Let $\left(\Lambda_{2}, \partial \Lambda_{2}\right)$ be the complementary pair. Then $\operatorname{Int}\left(\Lambda_{2}\right)=[v, \widehat{1})$. We also denote the corresponding order complexes $\Delta=\mathcal{O}(\Lambda), \Delta_{1}=\mathcal{O}\left(\Lambda_{1}\right), \Delta_{2}=\mathcal{O}\left(\Lambda_{2}\right), \partial \Delta_{1}=$ $\partial \Delta_{2}=\mathcal{O}\left(\partial \Delta_{1}\right)$.

Consider the exact sequence.

$$
0 \longrightarrow \mathbb{R}_{\Delta_{1}} \longrightarrow \mathbb{R}_{\Delta} \longrightarrow \mathbb{R}_{\operatorname{Int}\left(\Delta_{2}\right)} \longrightarrow 0
$$

Suppose we know that $\mathbb{R}_{\operatorname{Int}\left(\Delta_{2}\right)}$ is Cohen-Macaulay, with the dual sheaf $\mathbb{R}_{\operatorname{Int}\left(\Delta_{2}\right)}^{\vee}$ having the same stalks as $\mathbb{R}_{\Delta_{2}}$, and that the dual map $\alpha: \mathbb{R}_{\operatorname{Int}\left(\Delta_{2}\right)}^{\vee} \rightarrow \mathbb{R}_{\Delta}^{\vee}$ is injective. Then all three sheaves are Cohen-Macaulay and we get the dual exact sequence

$$
0 \longleftarrow \mathbb{R}_{\Delta_{1}}^{\vee} \longleftarrow \mathbb{R}_{\Delta} \stackrel{\alpha}{\longleftarrow} \mathbb{R}_{\operatorname{Int}\left(\Delta_{2}\right)}^{\vee} \longleftarrow 0
$$

The sheaf $\mathbb{R}_{\text {Int }\left(\Delta_{2}\right)}^{\vee}$ being a subsheaf of the constant sheaf must be the constant sheaf $\mathbb{R}_{\Delta_{2}}$. It follows from this that both $\Delta_{1}$ and $\Delta_{2}$ are near-Gorenstein, hence $\Lambda_{1}$ and $\Lambda_{2}$ are near-Gorenstein*.

Let us take $x \in \Delta_{2}$. We need to show that the stalk $\mathbb{R}_{\operatorname{Int}\left(\Delta_{2}\right), x}^{\vee}$ is well-defined (no higher cohomology in the cellular complex), one-dimensional, and that it injects into $\mathbb{R}_{\Delta, x}^{\vee}=\mathbb{R}$ by the map $\alpha$.

First consider the case when $x \in \operatorname{Int}\left(\Delta_{2}\right)$. Then the entire interval $[x, \widehat{1})$ in $\Delta$ lies in $\operatorname{Int}\left(\Delta_{2}\right)$. The two complexes that compute the stalks of $\mathbb{R}_{\Delta, x}^{\vee}$ and $\mathbb{R}_{\operatorname{Int}\left(\Delta_{2}\right), x}^{\vee}$ are equal, with the map between them being the identity.

Second consider the case when $x \in \partial \Delta_{2}$. Then $x$ is a chain

$$
x=\left\{\widehat{0}<\sigma_{1}<\sigma_{2}<\cdots<\sigma_{k}\right\}
$$


where $\sigma_{i} \vee v<\widehat{1}, \sigma_{i} \notin[v, \widehat{1})$ for all $i$. Let

$$
\Pi=[x, \widehat{1}) \cap \operatorname{Int}\left(\Delta_{2}\right) .
$$

An element of $\Pi$ is a refinement of $x$ that contains at least one $\tau \in \operatorname{Int}\left(\Lambda_{2}\right)=[v, \widehat{1})$. Such a $\tau$ must necessarily satisfy $\sigma_{k}<\tau$. If we let $S=\left[\sigma_{k} \vee v, \widehat{1}\right)$, then we have

$$
\Pi=\bigcup_{\tau \in S}\left[\left\{\widehat{0}<\sigma_{1}<\cdots<\sigma_{k}<\tau\right\}, \widehat{1}\right)
$$

It remains to prove the cellular complex of $\mathbb{R}_{\Pi}$ has one-dimensional cohomology in degree 0 and that the projection map $\mathbb{R}_{[x, \widehat{1})} \rightarrow \mathbb{R}_{\Pi}$ induces a surjection in the cohomology.

Consider the complex of sheaves indexed by chains in $S$ where the maps are the projections with \pm signs:

$$
\mathbb{R}_{\Pi} \longrightarrow \bigoplus_{\sigma_{k} \vee v \leq \tau} \mathbb{R}_{[\{x<\tau\}, \widehat{1})} \longrightarrow \bigoplus_{\sigma_{k} \vee v \leq \tau_{1}<\tau_{2}} \mathbb{R}_{\left[\left\{x<\tau_{1}<\tau_{2}\right\}, \widehat{1}\right)} \longrightarrow \cdots
$$

We claim that this gives a resolution of $\mathbb{R}_{\Pi}$. Indeed, if $\pi \in \Pi$ is a chain that contains $\left\{\tau_{1}<\tau_{2}<\cdots<\tau_{l}\right\}$, where $\tau_{i} \in S$, then the stalk of this complex at $\pi$ is indexed by subsets of $\left\{\tau_{1}, \ldots, \tau_{l}\right\}$, hence is acyclic.

We can compute the cellular cohomology of $\mathbb{R}_{\Pi}$ by a spectral sequence, where we first compute the cellular cohomology of each sheaf in the resolution. All sheaves in the complex have only degree zero cohomology of dimension one because $\Delta$ is Gorenstein. This implies that the cellular cohomology of $\mathbb{R}_{\Pi}$ is computed by the complex

$$
\bigoplus_{\sigma_{k} \vee \nu \leq \tau} \mathbb{R} \longrightarrow \bigoplus_{\sigma_{k} \vee \nu \leq \tau_{1}<\tau_{2}} \mathbb{R} \longrightarrow \cdots
$$

Note that if we indexed the terms in this complex by chains $\tau_{1}<\tau_{2}<\cdots$, where $\tau_{1}$ is strictly greater than $\sigma_{k} \vee v$, then we would get the cellular complex of $\mathcal{O}\left(\left[\sigma_{k} \vee v, \widehat{1}\right)\right)$. Since we also allow $\tau_{1}=v$, we get the cellular complex of

$$
\mathcal{O}([v, \widehat{1})) \times\{\widehat{0}, \widehat{1}\}
$$

but without the minimal element $\widehat{0}$. The cellular complex of the product is acyclic and the missing minimal element gives us that the degree zero cohomology of the cellular complex of $\mathbb{R}_{\Pi}$ has dimension one.

To see that we have surjectivity of

$$
H^{0}\left(C^{\bullet}\left(\mathbb{R}_{[x, \widehat{1})}, \Delta\right)\right) \longrightarrow H^{0}\left(C^{\bullet}\left(\mathbb{R}_{\Pi}, \Delta\right)\right)
$$

note that we can make the complex (4.5) exact by extending it from the left by

$$
H^{0}\left(C^{\bullet}\left(\mathbb{R}_{[x, \widehat{1})}, \Delta\right)\right) \longrightarrow \bigoplus_{\sigma_{k} \vee v \leq \tau} \mathbb{R} \longrightarrow \cdots,
$$


which comes from extending the complex of sheaves

$$
\mathbb{R}_{[x, \widehat{1})} \longrightarrow \mathbb{R}_{\Pi} \longrightarrow \bigoplus_{\sigma_{k} \vee v \leq \tau} \mathbb{R}_{[\{x<\tau\}, \widehat{1})} \longrightarrow \cdots
$$

This finishes the proof of Lemma 2.10 and Theorem 1.2.

\section{Appendix}

The purpose of this appendix is to prove non-negativity of the cd-index for Gorenstein* and near-Gorenstein* posets (Theorems 2.4 and 2.5). The two theorems are proved in [10] for complete and quasi-convex fans, while the generalization to Gorenstein* posets is left largely to the reader. We give the necessary details here.

We start by recalling another construction of the cd-index in terms of two operations $\mathcal{C}$ and $\mathcal{D}$ on Cohen-Macaulay sheaves on a poset $\Pi$.

Let $\Pi$ be a rank $n$ poset and $\mathcal{O}(\Pi)$ its order complex. Define the map of posets:

$$
\begin{aligned}
\beta: & \mathcal{O}(\Pi) \\
\left\{0<\sigma_{1}<\cdots<\sigma_{k}\right\} & \longmapsto \sigma_{k}
\end{aligned}
$$

If $F$ is a sheaf on $\Pi$, the pullback $\beta^{*}(F)$ is a sheaf on $\mathcal{O}(\Pi)$ with stalks

$$
\beta^{*}(F)_{x}=F_{\beta(x)}
$$

and the obvious restriction maps.

Let $F$ be a sheaf on $\Pi$ with finite dimensional stalks. We define the ab-index of $F$ as follows (recall the definition of $\mathrm{wt}(x)$ in Section 2.2):

$$
\Psi_{F}=\sum_{x \in \mathcal{O}(\Pi)} \operatorname{wt}(x) \operatorname{dim} \beta^{*}(F)_{x}
$$

If this polynomial can be written in terms of variables $\mathbf{c}=\mathbf{a}+\mathbf{b}$ and $\mathbf{d}=\mathbf{a b}+\mathbf{b a}$, then we call the resulting cd-polynomial the cd-index of the sheaf $F$. It often happens that the ab-index can be expressed only in the form

$$
\Psi_{F}=f(\mathbf{c}, \mathbf{d})+g(\mathbf{c}, \mathbf{d}) \mathbf{a}
$$

where $f$ and $g$ are homogeneous cd-polynomials of degree $n$ and $n-1$, respectively.

Next we recall the proof of non-negativity of the cd-index in the case of fans. For fans one can define the cellular complex of a sheaf as in the case of simplicial complexes. With the cellular complex one gets the notion of Cohen-Macaulay sheaves and duality.

Let $\Pi$ be the poset of an $n$-dimensional but not necessarily complete fan and $F$ a Cohen-Macaulay sheaf on $\Pi$. We define two operations $\mathcal{C}$ and $\mathcal{D}$ that each produce another Cohen-Macaulay sheaf on $(n-1)$ - and $(n-2)$-dimensional fans, respectively.

The sheaf $\mathcal{C}(F)$ is simply the restriction of $F$ to the $(n-1)$-skeleton $\Pi^{\leq n-1}$ of $\Pi$. It is a Cohen-Macaulay sheaf on $\Pi^{\leq n-1}$. The sheaf $\mathcal{D}(F)$ is defined by choosing a $\underline{\text { Springer }}$ 
surjective map

$$
\alpha: C(F)^{\vee} \longrightarrow C(F)
$$

and setting $\mathcal{D}(F)$ equal to the kernel of $\alpha$. Since $C(F)^{\vee}$ and $C(F)$ have isomorphic stalks on cones of maximal dimension $n-1$, it follows that $\mathcal{D}(F)$ is a sheaf on the $(n-2)$-skeleton of $\Pi$. From the short-exact sequence of sheaves we get that $\mathcal{D}(F)$ is a Cohen-Macaulay sheaf on $\Pi \leq n-2$.

Since both $\mathcal{C}$ and $\mathcal{D}$ produce Cohen-Macaulay sheaves, we can compose them. If $w(\mathbf{c}, \mathbf{d})$ is a monomial in $\mathbf{c}$ and $\mathbf{d}$ of degree $m$, then applying $w(\mathcal{C}, \mathcal{D})$ to the sheaf $F$, we get a sheaf on the $(n-m)$-skeleton of $\Pi$. In particular, if $m=n$, then $w(\mathcal{C}, \mathcal{D})(F)$ is a sheaf on the zero cone.

The following is the most general positivity result proved in [10]:

Theorem 5.1. Let $\Pi$ be an $n$-dimensional fan and $F$ a Cohen-Macaulay sheaf on $\Pi$, such that the ab-index of $F$ can be expressed in the form

$$
\Psi_{F}=f(\mathbf{c}, \mathbf{d})+g(\mathbf{c}, \mathbf{d}) \mathbf{a} .
$$

For any cd-monomial $w(\mathbf{c}, \mathbf{d})$ of degree $n$, the coefficient of $w$ in $f(\mathbf{c}, \mathbf{d})$ is equal to the dimension of the stalk

$$
w(\mathcal{C}, \mathcal{D})(F)_{0}
$$

In particular, $f(\mathbf{c}, \mathbf{d})$ has non-negative integer coefficients.

Applying this theorem to the constant sheaf $\mathbb{R}_{\Pi}$ on a complete or quasi-convex fan gives the non-negativity of the cd-index.

If, instead of sheaves on $\Pi$, we work with the dimensions of the stalks of such sheaves, then the operations $\mathcal{C}$ and $\mathcal{D}$ give a method for computing the coefficients of the cd-index. It is clear that the same method then works for an arbitrary Eulerian poset. However, we need sheaves to show non-negativity of the cd-index.

For an arbitrary poset, the main difficulty in extending the operations $\mathcal{C}$ and $\mathcal{D}$ above lies in the definition of the cellular complex and hence the dual sheaf. Recall that the maps in the cellular complex are the restrictions with appropriate signs. In the case of fans one gets a compatible set of signs by orienting each cone and setting the signs equal to \pm 1 depending on the orientations. For an arbitrary poset, there may not exist such a compatible set of orientations. Thus, we have to do all computations involving cellular complexes on the order complex $\mathcal{O}(\Pi)$.

For the rest of this appendix, let $\Pi$ be a poset of rank $n$. П need not be Gorenstein*. However, we require that for any $\sigma \in \Pi$ the subposet $[\widehat{0}, \sigma)$ be Gorenstein*. The only case we will need is when $\Pi$ is the $n$-skeleton of a Gorenstein* poset of rank $\geq n$.

Definition 5.2. A sheaf $F$ on $\Pi$ is Cohen-Macaulay if $\beta^{*}(F)$ is Cohen-Macaulay on $\mathcal{O}(\Pi)$. 
Lemma 5.3. Let $F$ be a Cohen-Macaulay sheaf on $\Pi$. Then there exists a CohenMacaulay sheaf $F^{\vee}$ on $\Pi$ such that

$$
\beta^{*}(F)^{\vee} \simeq \beta^{*}\left(F^{\vee}\right)
$$

Proof: For $\sigma \in \Pi$, consider the fiber $\beta^{-1}(\sigma)$. This subposet of $\mathcal{O}(\Pi)$ has the minimal element $x=\{\widehat{0}<\sigma\}$. We show that for any $y \in \beta^{-1}(\sigma)$, the restriction map in the dual sheaf

$$
\beta^{*}(F)_{y}^{\vee} \longrightarrow \beta^{*}(F)_{x}^{\vee}
$$

is an isomorphism. We can then define

$$
F_{\sigma}^{\vee}=\beta^{*}(F)_{x}^{\vee}
$$

and restriction maps for $\sigma>\tau$

$$
F_{\sigma}^{\vee}=\beta^{*}(F)_{\left\{\widehat{0}_{<\sigma\}}\right.}^{\vee} \simeq \beta^{*}(F)_{\{\widehat{0}<\tau<\sigma\}}^{\vee} \longrightarrow \beta^{*}(F)_{\left\{\widehat{0}_{<\tau\}}\right.}^{\vee}=F_{\tau}^{\vee}
$$

These maps are compatible and define the sheaf $F^{\vee}$. It is also easy to check that this $F^{\vee}$ satisfies the isomorphism in the statement of the lemma.

To prove the isomorphism (5.6), note that the stalks of the dual sheaf are computed by the cellular complexes over the intervals $[y, \widehat{1})$ and $[x, \widehat{1})$, respectively. We write $[x, \widehat{1})$ as a product of two posets

$$
[x, \widehat{1})=S_{x} \times T,
$$

where $S_{x}$ consists of all refinements of the chain $x$ with last element $\sigma$ and $T$ consists of all the chains between $\sigma$ and $\widehat{1}$. Similarly, write

$$
[y, \widehat{1})=S_{y} \times T .
$$

Since the index set of each cellular complex is a product of two posets, we can write the complex as a double complex and compute its cohomology first along rows and then along columns. Thus, we have two double complexes and a map between them. For a fixed $t=\left\{\widehat{0}<\tau_{1}<\cdots<\tau_{k}\right\} \in T$, the rows of the double complexes indexed by $S_{x}$ and $S_{y}$, respectively, are the cellular complexes computing the dual of the constant sheaf with stalk $F_{\tau}$ on the poset $\mathcal{O}([0, \sigma))$. By Lemma 4.5, these two complexes have only degree zero cohomology isomorphic to $F_{\tau}$ and the map between the complexes induces an isomorphism of cohomologies. Thus, after taking cohomology along rows, we get two isomorphic complexes, indexed by the same set $T$. Clearly, the cohomologies of these complexes are also isomorphic. 


\section{Remark 5.4.}

(1) Observe that there is no choice involved in the definition of $F^{\vee}$ given in the proof of Lemma 5.3. In particular, $F \mapsto F^{\vee}$ is a contravariant functor on the category of Cohen-Macaulay sheaves on $\Pi$.

(2) It is easy to check that

$$
\operatorname{dim} F_{\sigma}^{\vee}=\sum_{\pi>\sigma}(-1)^{n-\rho(\pi)} \operatorname{dim} F_{\pi}
$$

Thus, at least numerically, we can think of $F^{\vee}$ as computed by a cellular complex on $\Pi$, similarly to the case of fans.

Let us now turn to the construction of the operations $\mathcal{C}$ and $\mathcal{D}$. For $F$ a sheaf on $\Pi$, let $\mathcal{C}(F)$ be its restriction to the $(n-1)$-skeleton of $\Pi$.

Lemma 5.5. If $F$ is a Cohen-Macaulay sheaf on $\Pi$, then $\mathcal{C}(F)$ is Cohen-Macaulay on $\Pi^{\leq n-1}$.

Proof: On $\Pi$ we have an exact sequence of sheaves

$$
0 \longrightarrow \mathcal{C}(F) \longrightarrow F \longrightarrow \bigoplus_{\rho(\sigma)=n} F_{\sigma} \longrightarrow 0
$$

where $F_{\sigma}$ is the constant sheaf with the stalk $F_{\sigma}$ supported on the single element $\sigma$. The pullback of this sequence to $\mathcal{O}(\Pi)$ is also exact. Now $\beta^{*}(F)$ is Cohen-Macaulay on $\mathcal{O}(\Pi)$ by assumption. Each sheaf $\beta^{*}\left(F_{\sigma}\right)$ is a constant sheaf on $[\{\widehat{0}<\sigma\}, \widehat{1}) \subset \mathcal{O}(\Pi)$. Since this subcomplex of $\mathcal{O}(\Pi)$ is Gorenstein, the constant sheaf is Cohen-Macaulay. From the exact sequence we get that $\mathcal{C}(F)$ is Cohen-Macaulay on $\Pi^{\leq n-1}$.

To define the sheaf $\mathcal{D}(F)$, we find a surjective map

$$
\alpha: \mathcal{C}(F)^{\vee} \longrightarrow \mathcal{C}(F)
$$

and set $\mathcal{D}(F)$ equal to its kernel. Since $\alpha$ is surjective, $\mathcal{D}(F)$ is a Cohen-Macaulay sheaf on the $(n-2)$-skeleton of $\Pi$.

The construction of $\alpha$ proceeds as in the case of fans in [10]. Let $\sigma \in \Pi$ be an element of rank $n$ and $f \in F_{\sigma}$ a section. Then $f$ defines a map of sheaves

$$
\begin{aligned}
\phi_{f}: \mathbb{R}_{[\widehat{0}, \sigma]} & \longrightarrow F \\
1_{\sigma} & \longmapsto f_{\sigma} .
\end{aligned}
$$

Restricting to the $(n-1)$-skeleton of $\Pi$ we get a map

$$
\phi_{f}: \mathbb{R}_{[\hat{0}, \sigma)} \longrightarrow \mathcal{C}(F) .
$$


We claim that $\mathbb{R}_{[0, \sigma)}$ is a Cohen-Macaulay sheaf on $\Pi^{\leq n-1}$ with an isomorphism

$$
\mathbb{R}_{[\widehat{0}, \sigma)}^{\vee} \stackrel{\simeq}{\longrightarrow} \mathbb{R}_{[\widehat{0}, \sigma)}
$$

Indeed, the pullback of $\mathbb{R}_{[\widehat{0}, \sigma)}$ to $\mathcal{O}(\Pi)$ is the constant sheaf on

$$
\operatorname{Link}_{\mathcal{O}(\Pi)}(\{\widehat{0}<\sigma\}) \simeq \mathcal{O}([\widehat{0}, \sigma))
$$

and the poset $[\widehat{0}, \sigma)$ is Gorenstein*.

Since the duality is functorial, we get for each $f \in F_{\sigma}$ a map

$$
\alpha_{f}: \mathcal{C}(F)^{\vee} \stackrel{\phi_{f}^{\vee}}{\longrightarrow} \mathbb{R}_{[\widehat{0}, \sigma)}^{\vee} \stackrel{\simeq}{\longrightarrow} \mathbb{R}_{[\widehat{0}, \sigma)} \stackrel{\phi_{f}}{\longrightarrow} \mathcal{C}(F) .
$$

We claim that a Zariski general linear combination of $\alpha_{f}$ (over all $\sigma$ and a basis of sections $f \in F_{\sigma}$ ) is a surjective map $\alpha$. For Cohen-Macaulay sheaves, it suffices to check surjectivity on maximal elements of the poset only. On an element $\tau$ of rank $n-1$, the map $\alpha$ is a composition

$$
F_{\tau}^{*} \stackrel{\phi^{*}}{\longrightarrow} \bigoplus_{\sigma, f} \mathbb{R}^{*} \longrightarrow \bigoplus_{\sigma, f} \mathbb{R} \stackrel{\phi}{\longrightarrow} F_{\tau}
$$

Since the rightmost map $\phi$ is surjective, it is clear that for a general diagonal map $\oplus \mathbb{R}^{*} \longrightarrow \oplus \mathbb{R}$, the composition is also surjective.

From these two operations $\mathcal{C}$ and $\mathcal{D}$ we get as in the case of fans:

Theorem 5.6. Let $\Pi$ be a rank $n$ poset such that for every $\sigma \in \Pi$ the interval $[\widehat{0}, \sigma)$ is Gorenstein*. Let $F$ be a Cohen-Macaulay sheaf on $\Pi$ such that its $\mathbf{a b - i n d e x ~ c a n ~ b e ~}$ expressed in the form

$$
\Psi_{F}=f(\mathbf{c}, \mathbf{d})+g(\mathbf{c}, \mathbf{d}) \mathbf{a} .
$$

Then for any cd-monomial $w(\mathbf{c}, \mathbf{d})$ of degree $n$, the coefficient of $w$ in $f(\mathbf{c}, \mathbf{d})$ is equal to the dimension of the stalk

$$
w(\mathcal{C}, \mathcal{D})(F)_{0}
$$

In particular, $f(\mathbf{c}, \mathbf{d})$ has non-negative integer coefficients.

Applying this theorem to the constant sheaf $\mathbb{R}_{\Pi}$ on a Gorenstein* poset $\Pi$ gives Theorem 2.4. To prove Theorem 2.5, we first apply the theorem to the constant sheaf $\mathbb{R}_{\Pi}$ on a near-Gorenstein* poset $\Pi$ to get non-negativity of $\Phi_{\Pi}(\mathbf{c}, \mathbf{d})$; since $\partial \Pi$ is

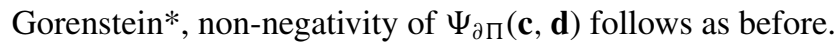

Acknowledgments The first author thanks Mittag-Leffler Institute where part of this research was carried out. He was also supported by NSF Grant DMS-0200624. The second author was supported by NSERC grant RGPIN 283301. 


\section{References}

1. K. Baclawski, "Cohen-Macaulay ordered sets,” J. Algebra 63 (1980), 226-258.

2. G. Barthel, J.-P. Brasselet, K.-H. Fieseler, and L. Kaup, "Combinatorial intersection cohomology for fans," Tohoku Math. J. 54 (2002), 1-41.

3. M.M. Bayer and L.J. Billera, "Generalized Dehn-Sommerville relations for polytopes, spheres and Eulerian partially ordered sets," Invent. Math. 79(1) (1985), 143-157.

4. M.M. Bayer and A. Klapper, "A new index for polytopes," Discrete Comput. Geom. 6 (1991), 33-47.

5. L.J. Billera and R. Ehrenborg, "Monotonicity of the cd-index for polytopes," Math. Z. 233 (2000), $421-441$.

6. T. Braden and R. MacPherson, "Intersection homology of toric varieties and a conjecture of Kalai," Comment Math. Helv. 74 (1999), 442-455.

7. P. Bressler and V.A. Lunts, "Intersection cohomology on nonrational polytopes," Compositio Math. 135(3) (2003), 245-278.

8. R. Ehrenborg and M. Readdy, "Coproducts and the cd-index," J. Algebraic Combin. 8 (1998), 273-299.

9. K. Karu, "Hard Lefschetz theorem for nonrational polytopes," Invent. Math. 157(2) (2004), 419-447.

10. K. Karu, "The cd-index of fans and posets," Compositio Math. 142 (2006), 701-718.

11. R.P. Stanley, Enumerative Combinatorics, vol. 1, Cambridge Studies in Advanced Mathematics, 49, Cambridge University Press, Cambridge, 1997.

12. R.P. Stanley, "Flag f-vectors and the cd-index," Math. Z. 216 (1994), 483-499.

13. R.P. Stanley, Combinatorics and Commutative Algebra, 2nd edition, Progress in Mathematics, vol. 41, Boston Basel Berlin: Birkhäuser, 1996. 